\title{
Quantum-Corrected Plasmonic Field Analysis Using a Time Domain PMCHWT Integral Equation
}

\author{
Ismail E. Uysal ${ }^{1}$, H. Arda Ulku ${ }^{1,2}$, and Hakan Bagci ${ }^{1}$ \\ ${ }^{1}$ Computer, Electrical and Mathematical Sciences and Engineering \\ King Abdullah University of Science and Technology (KAUST), Thuwal, Jeddah, 23955-6900, Saudi Arabia \\ e-mails: \{ismail.uysal, huseyin.ulku, hakan.bagci\}@kaust.edu.sa \\ ${ }^{2}$ Electronics Engineering, Gebze Technical University, Gebze, Kocaeli, 41400, Turkey, e-mail: haulku@gtu.edu.tr
}

\begin{abstract}
When two structures are within sub-nanometer distance of each other, quantum tunneling, i.e., electrons "jumping" from one structure to another, becomes relevant. Classical electromagnetic solvers do not directly account for this additional path of current. In this work, an auxiliary tunnel made of Drude material is used to "connect" the structures as a support for this current path (R. Esteban et al., Nat. Commun., 2012). The plasmonic fields on the resulting connected structure are analyzed using a time domain surface integral equation solver. Time domain samples of the dispersive medium Green function and the dielectric permittivities are computed from the analytical inverse Fourier transform applied to the rational function representation of their frequency domain samples.
\end{abstract}

\section{INTRODUCTION}

Plasmonic field interactions on nanostructures are often analyzed by numerical schemes developed for solving Maxwell equations. However, when two structures are located within a sub-nanometer distance of each other, quantum tunneling effects become relevant; and the additional current path generated by the electrons "jumping" from one structure to another cannot be accounted for using these classical electromagnetic solvers. To overcome this problem, a quantum-corrected model has been proposed in [1]. This model introduces an auxiliary tunnel with material properties obtained using quantum models to "connect" the two nanostructures. The fields on the resulting connected structure can be analyzed using a classical electromagnetic solver.

In this work, this quantum correction scheme is incorporated within a time domain surface integral equation solver (TDSIE) to accurately analyze transient plasmonic field interactions on nanostructures. More specifically, a marching onin-time (MOT) scheme is developed to solve the PoggioMiller-Chan-Harrington-Wu-Tsai (PMCHWT) SIE [2], which is enforced on the interfaces between different dielectric volumes representing the nanostructures and the auxiliary tunnel. The dispersive dielectric permittivities of these volumes are represented using Drude models with different parameters. Parameters relevant to tunnel material are computed from one dimensional quantum characterization of the gap [1]. This scheme calls for computation of the time domain samples of the Green functions of the unbounded media with the material properties of each volume and their permittivities. These samples are obtained by inverse Fourier transforming the rational functions fitted to the frequency domain samples using the fast relaxed vector fitting (FRVF) algorithm [3]. Computation of the double temporal convolutions involving these time samples, which are called for by the MOT scheme, is carried out using the technique described in [4].

\section{FORMULATION}

Let $V_{m}, m=1,2, \ldots, M$, denote the volumes of $M$ scatterers residing in unbounded volume $V_{0}$. The permittivity and permeability of $V_{m}$ are $\varepsilon_{m}(t)$ and $\mu_{m} . V_{m}$ are bounded by surfaces $S_{l}, l=1,2, \ldots, L$. On each surface, two boundary conditions are enforced

$$
\begin{gathered}
\hat{\mathbf{n}}_{l m}(\mathbf{r}) \times\left.\left[\partial_{t} \mathbf{E}_{m}^{i n c}(\mathbf{r}, t)-\partial_{t} \mathbf{E}_{n}^{i n c}(\mathbf{r}, t)\right]\right|_{\mathbf{r} \in S_{l}}= \\
-\hat{\mathbf{n}}_{l m}(\mathbf{r}) \times\left.\left[\partial_{t} \mathbf{E}_{m}^{s c a}(\mathbf{r}, t)-\partial_{t} \mathbf{E}_{n}^{s c a}(\mathbf{r}, t)\right]\right|_{\mathbf{r} \in S_{l}} \\
\hat{\mathbf{n}}_{l m}(\mathbf{r}) \times\left.\left[\partial_{t} \mathbf{H}_{m}^{i n c}(\mathbf{r}, t)-\partial_{t} \mathbf{H}_{n}^{i n c}(\mathbf{r}, t)\right]\right|_{\mathbf{r} \in S_{l}}= \\
-\hat{\mathbf{n}}_{l m}(\mathbf{r}) \times\left.\left[\partial_{t} \mathbf{H}_{m}^{s c a}(\mathbf{r}, t)-\partial_{t} \mathbf{H}_{n}^{s c a}(\mathbf{r}, t)\right]\right|_{\mathbf{r} \in S_{l}} .
\end{gathered}
$$

Here, $\hat{\mathbf{n}}_{l m}(\mathbf{r})$ is unit normal vector on $S_{l}$ pointing towards $V_{m}$, and $\left\{\mathbf{E}_{m}^{i n c}(\mathbf{r}, t), \mathbf{H}_{m}^{i n c}(\mathbf{r}, t)\right\}$ and $\left\{\mathbf{E}_{m}^{s c a}(\mathbf{r}, t), \mathbf{H}_{m}^{s c a}(\mathbf{r}, t)\right\}$ represent incident and scattered fields in $V_{m}$. PMCHWT SIE is obtained by expressing $\mathbf{E}_{m}^{s c a}(\mathbf{r}, t)$ and $\mathbf{H}_{m}^{s c a}(\mathbf{r}, t)$ in (1) and (2), in terms of equivalent surface electric and magnetic current densities, $\mathbf{J}_{l}(\mathbf{r}, t)$ and $\mathbf{M}_{l}(\mathbf{r}, t)[2]$ :

$$
\begin{aligned}
\partial_{t} \mathbf{E}_{m}^{s c a}(\mathbf{r}, t) & =\sum_{l^{\prime}}\left[\mathcal{L}_{m}\left\{\mu_{m} \mathbf{J}_{l^{\prime}}(\mathbf{r}, t)\right\}\right. \\
& \left.-\mathcal{Q}_{m}\left\{\bar{\varepsilon}_{m}(t) * \mathbf{J}_{l^{\prime}}(\mathbf{r}, t)\right\}+\mathcal{K}_{m}\left\{\mathbf{M}_{l^{\prime}}(\mathbf{r}, t)\right\}\right] \\
\partial_{t} \mathbf{H}_{m}^{s c a}(\mathbf{r}, t) & =\sum_{l^{\prime}}\left[\mathcal{L}_{m}\left\{\varepsilon_{m}(t) * \mathbf{M}_{l^{\prime}}(\mathbf{r}, t)\right\}\right. \\
& \left.-\mathcal{Q}_{m}\left\{\mu_{m}^{-1} \mathbf{M}_{l^{\prime}}(\mathbf{r}, t)\right\}-\mathcal{K}_{m}\left\{\mathbf{J}_{l^{\prime}}(\mathbf{r}, t)\right\}\right]
\end{aligned}
$$

Here, the summation index $l^{\prime}$ runs over the surfaces that bounds $V_{m}$, “*” denotes temporal convolution, and integral operators are defined as

$$
\begin{aligned}
\mathcal{L}_{m}\left\{\mathbf{X}_{l^{\prime}}(\mathbf{r}, t)\right\} & =\int_{S_{l^{\prime}}} G_{m}(R, t) * \partial_{t}^{2} \mathbf{X}_{l^{\prime}}\left(\mathbf{r}^{\prime}, t\right) d \mathbf{r}^{\prime} \\
\mathcal{Q}_{m}\left\{\mathbf{X}_{l^{\prime}}(\mathbf{r}, t)\right\} & =\nabla \int_{S_{l^{\prime}}} G_{m}(R, t) * \nabla^{\prime} \cdot \mathbf{X}_{l^{\prime}}\left(\mathbf{r}^{\prime}, t\right) d \mathbf{r}^{\prime} \\
\mathcal{K}_{m}\left\{\mathbf{X}_{l^{\prime}}(\mathbf{r}, t)\right\} & =\nabla \times \int_{S_{l^{\prime}}} G_{m}(R, t) * \partial_{t} \mathbf{X}_{l^{\prime}}\left(\mathbf{r}^{\prime}, t\right) d \mathbf{r}^{\prime} .
\end{aligned}
$$

In (5), $R=\left|\mathbf{r}-\mathbf{r}^{\prime}\right|, G_{m}(R, t)$ is Green function of the unbounded medium with permittivity $\varepsilon_{m}(t)$ and permeability $\mu_{m}$. Equivalent surface current densities on each surface $S_{l}$ are 
approximated using the Rao-Wilton-Glisson (RWG) functions $\mathbf{f}_{n}^{l}(\mathbf{r})$ and Lagrange interpolation functions $T(t)[2]$ :

$$
\begin{aligned}
& \mathbf{J}_{l}(\mathbf{r}, t)=\sum_{j=1}^{N_{t}} \sum_{n=1}^{N_{s}^{l}} J_{j n}^{l} T(t-j \Delta t) \mathbf{f}_{n}^{l}(\mathbf{r}) \\
& \mathbf{M}_{l}(\mathbf{r}, t)=\sum_{j=1}^{N_{t}} \sum_{n=1}^{N_{s}^{l}} M_{j n}^{l} T(t-j \Delta t) \mathbf{f}_{n}^{l}(\mathbf{r}) .
\end{aligned}
$$

Here, $\Delta t$ is time step size, $N_{t}$ is number of time steps, and $N_{s}^{l}$ is the number of RWG basis functions on surface $S_{l}$, and $J_{j n}^{l}$ and $M_{j n}^{l}$ are the unknown current coefficients. Inserting (6)-(7), and (3)-(4) into (1)-(2), and testing the resulting equations in space and time using $\left.\left\langle\mathbf{f}_{m}(\mathbf{r}) \cdot \mathbf{F}(\mathbf{r}, t)\right\rangle\right|_{t=i \Delta t}=$ $\int \delta(t-i \Delta t) d t \int \mathbf{f}_{m}(\mathbf{r}) \cdot \mathbf{F}(\mathbf{r}, t) d \mathbf{r}$ yield [2]

$$
\mathbf{Z}_{0} \mathbf{I}_{i}=\mathbf{V}_{i}-\sum_{j=1}^{i-1} \mathbf{Z}_{i-j} \mathbf{I}_{j}
$$

Here, $\mathbf{I}_{i}$ and $\mathbf{V}_{i}$ store the unknown coefficients and the tested incident fields, and $\mathbf{Z}_{i-j}$ are the MOT matrices, respectively. $\mathbf{I}_{i}$ is solved using the well-known MOT scheme [2]. The elements of $\mathbf{Z}_{i-j}$ call for the computation of a double temporal convolution [see the terms $\mathcal{Q}_{m}\left\{\bar{\varepsilon}_{m}(t) * \mathbf{J}_{l^{\prime}}(\mathbf{r}, t)\right\}$ and $\mathcal{L}_{m}\left\{\varepsilon_{m}(t) * \mathbf{M}_{l^{\prime}}(\mathbf{r}, t)\right\}$ in (3)], which is carried out efficiently using the method proposed in [4]. It is assumed that all $V_{m}$ are non-magnetic, i.e., $\mu_{m}=\mu_{0}$. For $V_{0}, \varepsilon_{0}(t)=\varepsilon_{0}$, $\bar{\varepsilon}_{0}(t)=1 / \varepsilon_{0}$ and $G_{0}(R, t)=\delta\left(t-R / c_{0}\right) / 4 \pi R$, where $\delta(\cdot)$ is the Dirac delta function and $c_{0}=1 / \sqrt{\varepsilon_{0} \mu_{0}}$ is the speed of light. Consequently, temporal convolutions in (3) and (4) can be evaluated analytically for $V_{0}$ [2]. On the other hand, for $m=1,2, \ldots, M$, analytical expressions of $\varepsilon_{m}(t), \bar{\varepsilon}_{m}(t)$, and $G_{m}(R, t)$ [and also $\partial_{R} G_{m}(R, t)$ ] do not exist. Therefore, FRVF [3] algorithm is applied to the frequency domain samples of $\varepsilon_{m}(\omega), \bar{\varepsilon}_{m}(\omega)=1 / \varepsilon_{m}(\omega)$, $G_{m}(R, \omega)=e^{-j \omega R \sqrt{\varepsilon_{m}(\omega) \mu_{m}}} / 4 \pi R$, and $\partial_{R} G_{m}(R, \omega)$. FRVF approximates each of these quantities using the expansion

$$
F(\omega) \approx d+j \omega f+\sum_{k=1}^{N}\left\{b_{k} /\left[j \omega+a_{k}\right]\right\}
$$

where $d$ and $f$ are real constants, $a_{k}$ and $b_{k}$ are poles and residues associated with the rational function, and $N$ is the number of poles. Positive values of $a_{k}$ are enforced while expressing the inverse Fourier transform of (9) as

$$
\mathcal{F}^{-1}\{F(\omega)\} \approx d \delta(t)+f \delta^{\prime}(t)+\sum_{k=1}^{N} b_{k} u(t) e^{-a_{k} t} .
$$

Here, $\delta^{\prime}($.$) is the first derivative of Dirac delta function and$ $u($.$) is the unit step function. Samples of \varepsilon_{m}(t), \bar{\varepsilon}_{m}(t)$, $G_{m}(R, t)$, and $\partial_{R} G_{m}(R, t)$ are computed using (10). It should be emphasized here that the method described above to compute these samples has allowed for the first time to use TD-SIEs in analyzing transient scattering from objects with dispersive material properties.

\section{NumericAl Results}

Scattering from two sodium dimers, one with the tunnel and one without is compared. The dimers are aligned in the $\hat{\mathbf{z}}$ direction, the radius of the spheres is $2.17 \mathrm{~nm}$, the shortest distance between them is $0.265 \mathrm{~nm}$, and the radius of the tunnel is 0.23

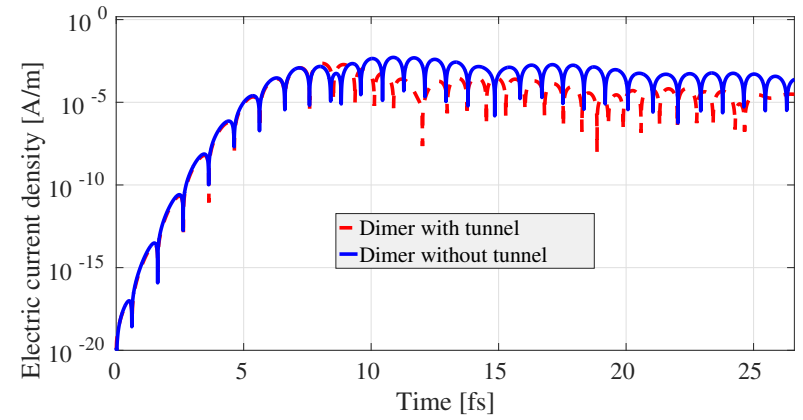

Fig. 1. Coefficient of the transient electric current density.

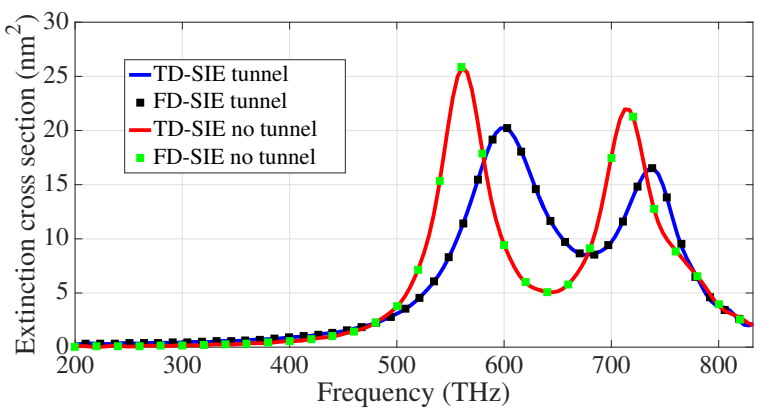

Fig. 2. Extinction cross section.

$\mathrm{nm}$. The permittivity of the spheres and tunnel is represented using the Drude model [1]. In both simulations, $\mathbf{E}^{\text {inc }}(\mathbf{r}, t)=$ $\hat{\mathbf{z}} g\left(t-t_{0}-\hat{\mathbf{x}} \cdot \mathbf{r} / c_{0}\right)$, where $g(t)=\cos \left(2 \pi f_{0} t\right) \exp \left(-t^{2} / 2 \sigma^{2}\right)$, $f_{0}=500 \mathrm{THz}, \sigma=3 /\left(2 \pi f_{0}\right)$, and $t_{0}=7.5 \sigma, N_{t}=4000$, $\Delta t=0.0067 \mathrm{fs}$, and $\mathbf{J}_{l}(\mathbf{r}, t)$ and $\mathbf{M}_{l}(\mathbf{r}, t)$ are discretized using 608 RWGs and 600 RWGs, respectively. Fig. 1 compares $J_{j n}^{l}$ for an RWG selected on the sphere surface. Fig. 2 compares extinction cross section obtained after Fourier transforming the proposed solver's currents to that directly computed by a frequency domain (FD)-SIE solver.

\section{CONCLUSION}

An MOT TD-PMCHWT-SIE solver is proposed for quantum-corrected analysis of plasmonic fields on nanostructures. The temporal samples (required by the MOT scheme) of the Green function and the permittivity of the dispersive materials of the structures and the auxiliary quantum tunnel are computed from the inverse Fourier transform applied to rational function representation (obtained by the FRVF algorithm) of their frequency domain samples.

\section{REFERENCES}

[1] R. Esteban, A. G. Borisov, P. Nordlander, and J. Aizpurua, "Bridging quantum and classical plasmonics with a quantum-corrected model," Nat. Comm., vol. 3, 825, 2012.

[2] B. Shanker, M. Lu, J. Yuan, and E. Michielssen, "Time domain integral equation analysis of scattering from composite bodies via exact evaluation of radiation fields," IEEE Trans. Antennas Propag., vol. 57, no. 5, pp. 1506-1519, 2009.

[3] B. Gustavsen and A. Semlyen, "Rational approximation of frequency domain responses by vector fitting," IEEE Trans. Power Del., vol. 14, no. 3, pp. 1052-1061, 1999.

[4] I. E. Uysal, H. A. Ulku, and H. Bagci, "MOT solution of the PMCHWT equation for analyzing transient scattering from conductive dielectrics," IEEE Antennas Wireless Propag. Lett., vol. 14, pp. 507-510, 2015. 